\title{
Mind the Microgap in Iridescent Cellulose Nanocrystal Films
}

\author{
Susete N. Fernandes, Pedro L. Almeida, Nuno Monge, Luis E. Aguirre, Dennys Reis, \\ Cristiano L. P. de Oliveira, António M. F. Neto, Pawel Pieranski, and Maria H. Godinho*
}

Solid films prepared from cellulose nanocrystals (CNCs) present remarkable optical properties: iridescence, ${ }^{[1]}$ selective reflection of left circularly polarized (LCP) light, and transmission of right circularly polarized (RCP) light. ${ }^{[2]}$ The same phenomenon has been observed in certain insect cuticles, for example in the Plusiotis batesi as well as in the Plusiotis optima, where only LCP light is selectively reflected by the cholesteric structure forming the outer exocuticle. ${ }^{[3]}$ In cellulosic films, the adopted left-handed chiral structure is attributed to the self-assembly of nanorods from chiral cellulose. ${ }^{[4]}$ The value of the wavelength of the reflected structure $(\lambda)$ depends upon the pitch $(P)$ and the average refraction index $(n)$ of the material according to the de Vries expression:

$\lambda=n P \sin \theta$

where $\theta$ is the angle between the incident light and the cholesteric layers. ${ }^{[5]}$ The tuning of the value of the cholesteric pitch

Dr. S. N. Fernandes, Prof. P. L. Almeida, N. Monge,

Dr. L. E. Aguirre, Prof. M. H. Godinho

i3N/CENIMAT

Department of Materials Science

Faculty of Science and Technology

Universidade NOVA de Lisboa

Campus de Caparica, 2829-516 Caparica, Portugal

E-mail:mhg@fct.unl.pt

Prof. P. L. Almeida

Área Departamental de Física

Instituto Superior de Engenharia de Lisboa

Instituto Politécnico de Lisboa

1959-007 Lisbon, Portugal

$\mathrm{N}$. Monge

CIAUD

Faculty of Architecture

University of Lisbon

Rua Sá Nogueira

Pólo Universitário

Alto da Ajuda

1349-055 Lisbon, Portugal

N. Monge

CIED

Lisbon School of Education

Polytechnic Institute of Lisbon

Campus de Benfica do IPL, 1549-003 Lisbon, Portugal

D. Reis, Prof. C. L. P. de Oliveira, Prof. A. M. F. Neto

Instituto de Física

Universidade de São Paulo

Rua do Matão, 1371, 05508-090 São Paulo, SP, Brazil

Prof. P. Pieranski

Laboratoire de Physique des Solides

UMR 8502

Université Paris-Sud

Bât. 510, 91405 Orsay, France

DOI: 10.1002/adma.201603560 can be at the origin of the cellulosic solid films' colors, which also varies with the viewing angle. Usually the solid films are prepared from isotropic or anisotropic suspensions of CNCs in water. The optical properties of the films were found to depend upon the characteristics of the colloidal suspensions, which pass through different stages upon solvent evaporation and before the formation of the film. In order to use these films for optical applications, many attempts have been undertaken to control their optical properties and mainly their reflection characteristics. ${ }^{[6-9]}$ If casting followed by a slow solvent-evaporation method $^{[4,8,10]}$ is used, the resulting films show iridescent colors and its cross-sections can present several domains with different pitch values. It has been found that the films' surface can have planar alignment over long distances, compared with the cellulose rods' lengths. ${ }^{[4]}$ However, the film's reflection peaks have broad widths indicating the presence of several pitch values, which should come from the cholesteric layers within the films. ${ }^{[11]}$ The existence of grooves between aligned layers has also been recently described in the literature. ${ }^{[12]}$ It seems that the evaporation of the water dictates the internal structure of the films, allowing the formation of several layers with different thicknesses and pitches. ${ }^{[10]}$ Some works have been devoted to avoid these defects. ${ }^{[8]}$

Other photonic structures that reflect both RCP and LCP light can be found in nature, as for example in the cuticle of Plusiotis resplendens. ${ }^{13]}$ The appearance of the insect is the result of a selective color reflection from a structure existing in its cuticle, consisting of an anisotropic domain, which acts as a half-wave phase retarder, sandwiched between two left-handed cholesteric layers. ${ }^{[3]}$ The reflected color depends on the pitch of the cholesteric structures, the average refractive index of the film and on the viewing angle, according to Equation (1).

Here, we report on a new cellulose-based photonic structure, which reflects both RCP and LCP light that can be tuned by temperature variation and the application of an external electric field. The optical properties of the CNC samples were investigated both in terms of their microstructure by scanning electron microscopy (SEM), small-angle X-ray scattering (SAXS) and spectroscopically, using circularly and linear polarized light coupled with a polarized optical microscope (POM).

The nanocrystals used in this work were prepared by acid hydrolysis of microcrystalline cellulose. Aqueous suspensions of CNCs $(\approx 1.9 \% \mathrm{w} / \mathrm{w})$ in the acid form were used $(\mathrm{pH}=2.9-3.5)$, which result in freestanding iridescent films after drying with colors depending on the viewing angle as described in the literature. ${ }^{[1,7,8]}$ The predominant colors of the film are green and orange when observed under unpolarized white light. These colors persist when a left polarizer is used (Figure S1, right hand side, Supporting Information) while the color vanishes for right polarized light (Figure S1, left hand 

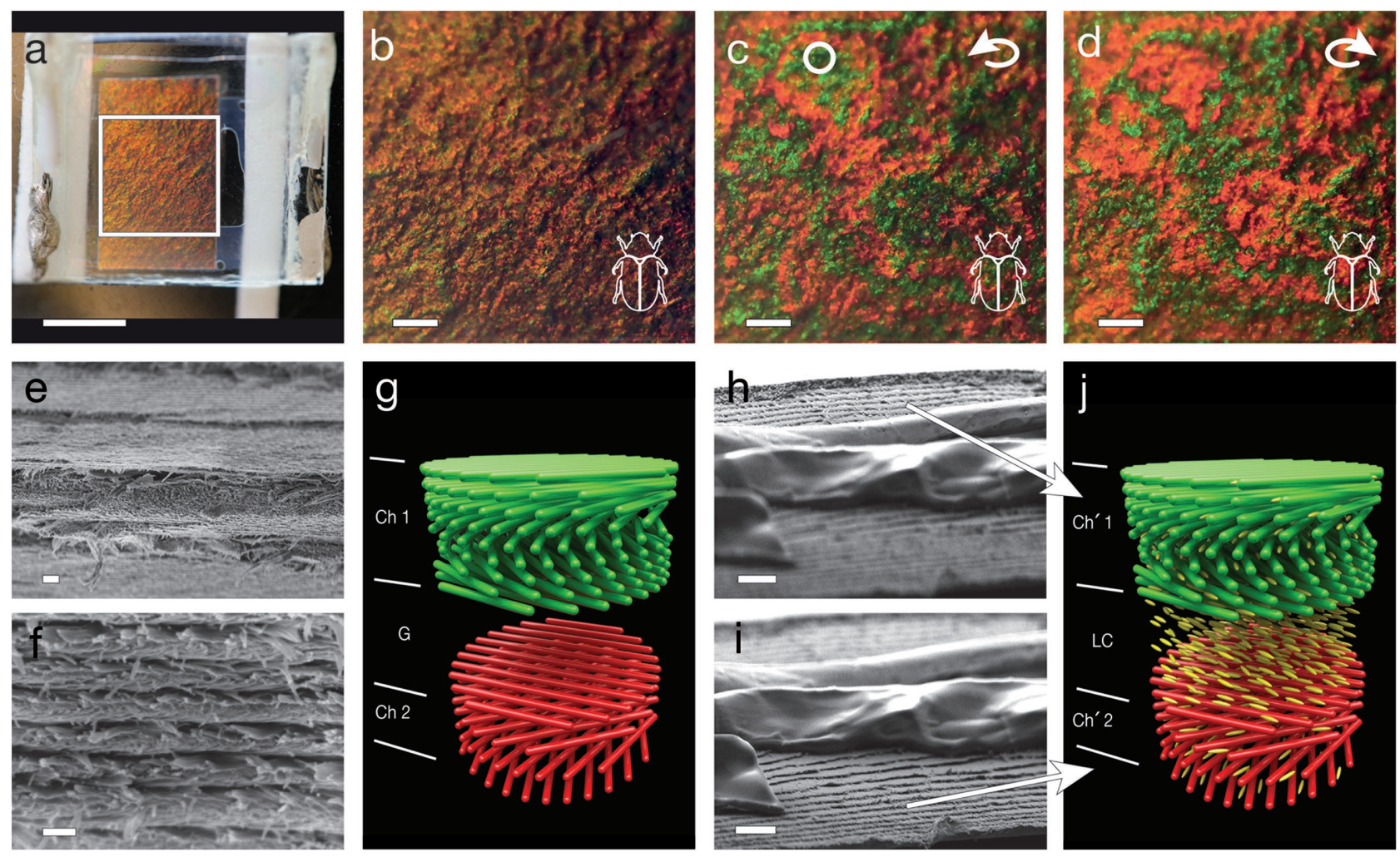

Figure 1. a-d) Photographs of CNC and 5CB composite film observed through unpolarized white light (a,b) and circularly polarized light showing different color reflection in LCP (c) and RCP (d) light channels as Plusiotis resplendens (silhouette in panels (b)-(d)). The circle marked in panel (c) represents the area of the sample analyzed in the upcoming figures. e) Details of a CNC chiral nematic film SEM image cross-section showing cholesteric regions separated by gaps. The structure in panel $(\mathrm{f})$ shows a detail of a cholesteric region with pitch, $173 \pm 15 \mathrm{~nm}$. The scheme (g) represents the cross-section near the gap, G, of a sandwich of two left cholesteric domains Ch1 and Ch2 with different pitches. h,i) SEM pictures, taken at room temperature, of the CNC film with the gap filled with an anisotropic liquid crystal (4CB), which is solid at room temperature, and infiltrated in the liquid-crystal state at $T=47.8^{\circ} \mathrm{C}$. j) Scheme showing the gap filled with the anisotropic layer sandwiched between the two left cholesteric domains (Ch'1 and Ch'2). Scale bars: a) $5 \mathrm{~mm}$; b,c,d) $1 \mathrm{~mm}$; e,f) $1 \mu \mathrm{m}$; h,i) $3 \mu \mathrm{m}$.

side, Supporting Information) indicating the presence of a left helicoidal structure. In accordance with the results obtained in the literature the handedness of the films is determined by the inherent chirality of the molecules that compose the cellulose nanocrystals and the film would be expected to present left handedness. ${ }^{[14]}$ The selective reflection of left circularly polarized light exhibited by the solid CNC films resembles the cuticles of certain beetles, for example the Plusiotis optima ${ }^{[15]}$ (inset silhouettes in the Figure S1 in the Supporting Information).

The iridescent CNCs solid films were then impregnated with 4-cyano-4'-pentylbiphenyl (5CB) nematic liquid crystal (LC), knowing that cellulose induces 5CB planar surface anchoring alignment. ${ }^{16]}$ Figure 1a,b shows a macroscopic photograph of the film under white light illumination; the predominant colors of the film are now red and green (see Movie S1, Supporting Information). The increase of the cellulose network cholesteric pitch is an indication that the CNC film was swollen by the liquid crystal. Moreover, this composite material reflects light under both left (Figure 1c) and right (Figure 1d) circular polarizers, mimicking the cuticle of Plusiotis resplendens (inset silhouettes in Figure $1 \mathrm{~b}-\mathrm{d}$ ). It is worth noting that the colors reflected by the film are not uniform; the red and green regions being the most predominant. The regions reflecting the green in the LCP channel become red in the RCP channel (and vice versa) (Figure 1c,d and Movie S1, Supporting Information). The layered films observed by SEM (Figure 1e and Figure S2d,e, Supporting Information) confirm the existence of the helical arrangement, allowing pitches' determination, as previously reported by other authors for cholesteric CNC phases. ${ }^{[11,14]}$ The film's cross-section shows domains with different pitches separated by gaps, which develop due to the cleavage stresses ${ }^{[17]}$ associated with the films anisotropy.

As was previously demonstrated, ${ }^{[18]}$ the cuticle of Plusiotis resplendens can be mimicked by sandwiching an anisotropic layer between two cholesteric regions with the same handedness and similar pitches and moreover an optical heterojunction anisotropic structure was also constructed by using cholesteric layers with different pitches. ${ }^{[19]}$ We wondered if a similar process could explain the CNC/5CB film optical characteristics.

SEM of solid films cross-sections revealed the presence of cholesteric layers separated by micrometer-sized gaps $(G)$, as in Figure 1e, schematically represented in Figure 1g. The cholesteric pitch varied from region to region in the top domain (Ch1) of the film, as well as in the cholesteric layers (Ch2) below the gaps. For our study we focused on a region (as indicated in Figure S1, circled region, in the Supporting Information) where 
the top cholesteric pitch in average Ch1 $=173 \pm 15 \mathrm{~nm}$ with a thickness of $2.6 \pm 0.2 \mu \mathrm{m}, \mathrm{Ch} 2=190 \pm 17 \mathrm{~nm}$ and thickness $3.0 \pm 0.2 \mu \mathrm{m}$, separated by a gap $G=0.7 \pm 0.1 \mu \mathrm{m}$. According to SAXS data (Figure S3, Supporting Information) the fractal dimension of the scatters is $D=2.9$ for the CNC film and $D$ $=2.5$ for the $\mathrm{CNC} / 5 \mathrm{CB}$ film, which indicates that the fractal aggregates show a $3 \mathrm{D}$ organization $(D=3)$. The CNC film sample has a fractal size $\approx 1000 \AA$ and this value increases by a factor of five to $\approx 5000 \AA$ for $\mathrm{CNC} / 5 \mathrm{CB}$ film corroborating the existence of the gaps as seen by SEM. According to the literature, ${ }^{[4]}$ we also found that the cholesteric order is conserved for micrometer-scale distances, much larger than the nanometric length of the CNC rods. The lateral size of the domains was found to be dependent upon many factors, which include the initial film precursor solutions' characteristics as well as the films' production conditions. ${ }^{[7,9,20]}$ The cross-section heterogeneities are in agreement with the literature: not only the existence of cholesteric planar layers with different pitches ${ }^{[8]}$ but also the micrometer gaps in between. ${ }^{[10]}$ These heterogeneities were essentially attributed to the capture of a variety of nonequilibrium states at different stages of water evaporation from the colloidal suspension ${ }^{[8]}$ which can be divided into several steps. ${ }^{[7,21]}$ The existence of gaps, also mentioned as cracks, was discussed in the literature and attributed to the anisotropic contraction of the chiral nematic gels during the formation of the films by casting-solvent evaporation. ${ }^{[10]}$

In this work we took advantage of the presence of these heterogeneities, in particular the existence of gaps, in order to understand and construct a new tunable thermal and electrooptical cellulose device. To ascertain the role of the liquid crystal, we started by infiltrating the mesomorphic compound, $4 \mathrm{CB}$, which is crystalline at room temperature, into the CNC film at $47.8{ }^{\circ} \mathrm{C}$. The composite material was then cooled to room temperature and examined by SEM microscopy. The SEM images of a cross-section of the film revealed the presence of a 4CB layer filling the gaps observed in the initial CNC solid films (Figure 1h,i; see Figure S2f-g, Supporting Information, for additional SEM images) between two left cholesteric domains (Ch'1 and Ch'2) with different pitches, $\mathrm{Ch}^{\prime} 1=224 \pm 48 \mathrm{~nm}$ and $\mathrm{Ch}^{\prime} 2=238 \pm 39 \mathrm{~nm}$, respectively. An idealized scheme of the cross-section of the film, showing the cholesteric domains and gap filled with a planar anchoring anisotropic layer, can be seen in Figure $1 j$.

To clarify the observed features of the original $\mathrm{CNC}$ and the composite $\mathrm{CNC} / 5 \mathrm{CB}$ films, POM observations as well as reflectance spectra were obtained for LCP and RCP channels, for normal incidence of light. The CNC film analyzed in the region indicated by the circle in Figure S1 in the Supporting Information displays an orange green color detected only in the LCP channel (Figure 2a), while the RCP reflection image is essentially colorless (Figure $2 \mathrm{~b}$ ), as expected for a left handed cholesteric structure. The broad band reflection LCP spectrum $(\Delta \lambda=130 \mathrm{~nm})$ reveals the presence of several left chiral nematic domains, existing in the bulk, with different pitch values centered at $\lambda=548 \mathrm{~nm}$ (Figure $2 \mathrm{f}$ ). In the scheme of Figure $2 \mathrm{~g}$, the different wavelengths reflected by the film are an indication of the left-handed chiral nematic domains, which are perpendicular to the film's cross-section.

The CNC/5CB film was found to reflect not only LCP light (Figure 2c), with maximum reflection centered in the green wavelength $(\lambda=518 \mathrm{~nm})$ (Figure $2 \mathrm{~h}, \mathrm{LCP}$ ) but also the RCP light was reflected (Figure $2 \mathrm{~d}$ ) with a maximum in the orangered wavelengths $(\lambda=610 \mathrm{~nm})$ (Figure $2 \mathrm{~h}, \mathrm{RCP})$. It is important to stress that a slight reflection at the blue wavelengths was observed for both light channels' spectra. The reflected image obtained between crossed polarizers (Figure 2e) denotes the
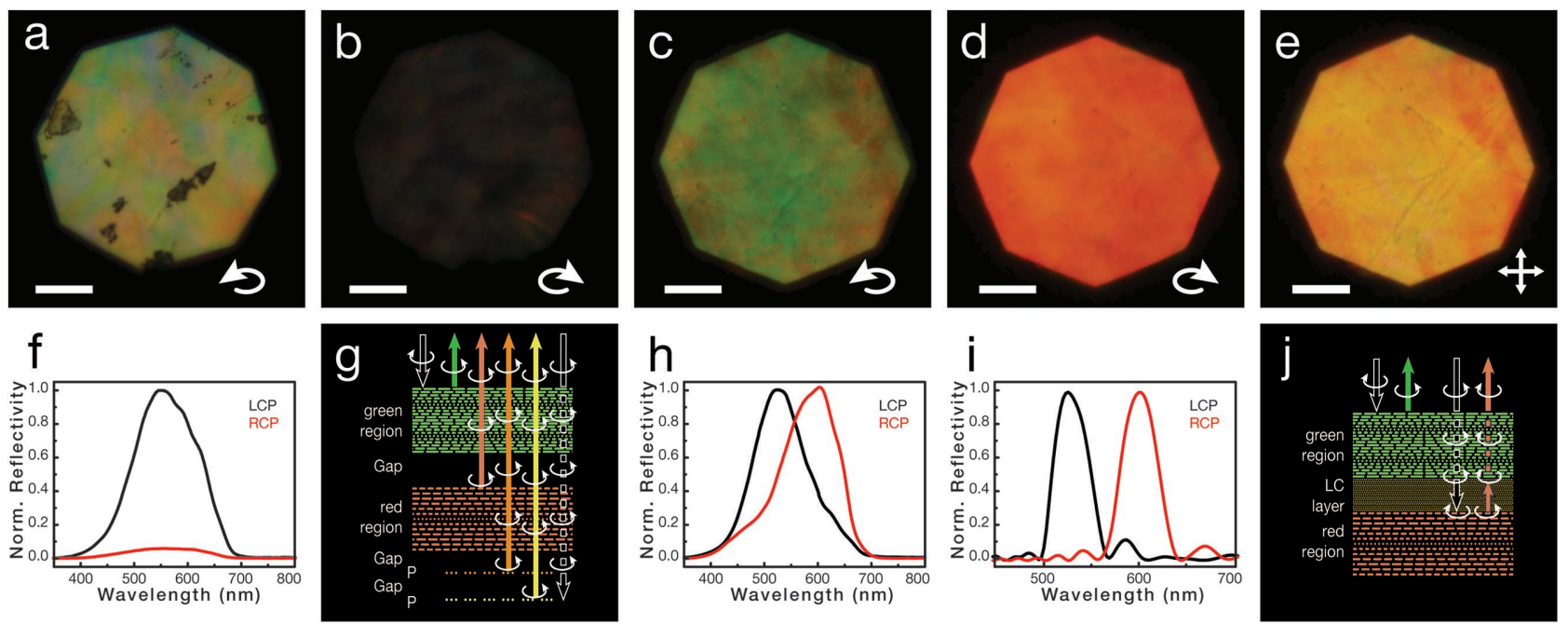

Figure 2. a,b) Optical polarized CNC films' reflection with no liquid crystal (the measurements were performed in the circle area shown in Figure S1 in the Supporting Information) - a) reflected LCP light and b) reflected RCP light. f) Reflectance spectra of the film for both channels LCP and RCP and g) scheme of the film layered structure showing reflection and transmittance optical characteristics. c-e) Film with LC (the optical images as well as the reflectance spectra were taken in the circled area in Figure 1c): c) reflected LCP light and d) reflected RCP light, e) the same area of the film between cross polarizers. The reflected circularly polarized components of the film with LC in panels (c) and (d) combine to give the color of the film as seen in panel (e). h,i) Spectra from LCP and RCP channels (h) and simulated reflectance spectra using Berreman's method (i). j) Scheme of the sandwiched structure, reflection, and transmittance optical characteristics of the film with a liquid-crystal layer acting as a $\lambda / 2$ plate. The scale bars represent $50 \mu \mathrm{m}$. 
superposition of the colors reflected by the two circular polarization channels. As the sense of the reflected circularly polarized light is directly related to the handedness of the cholesteric layer, these observations might denote that two overlaid cholesteric structures, with opposite chiralities, could be present in the reflecting $\mathrm{CNC} / 5 \mathrm{CB}$ layers, as suggested for the structural colors observed for Pollia fruit. ${ }^{[2]}$ This possibility is refuted if one takes into account the presence of the anisotropic-liquidcrystal layer. In fact, the anisotropic layer acts as a half-wave retardation plate for a given wavelength, $\lambda$, when the phase difference produced between the ordinary and extraordinary rays:

$$
\Delta \phi=\frac{\Delta n d}{\lambda} 2 \pi
$$

matches $\pi$ ( $\Delta n$ is the birefringence and $d$ is the thickness of the anisotropic layer). ${ }^{[3,23]}$ Taking into account the birefringence of the 5CB liquid crystal, $\Delta n=0.3$, and the thickness of the gaps' measured by SEM in the presence of LC, $d=870 \pm 90 \mathrm{~nm}$, one finds that the nematic layer works fairly well as a $\lambda / 2$ retardation plate within the wavelength interval of $370-650 \mathrm{~nm}$. The scheme shown in Figure $2 j$ illustrates the $\lambda / 2$ retardation plate's role between the two left cholesteric domains with different pitches when circularly polarized light is incident perpendicularly to the film. The light with left-handed circular polarization is completed reflected by the green cholesteric domain, $\lambda_{0} \approx$ $520 \mathrm{~nm}$, located at the top of the film while the right-handed circular polarization penetrates it without any loss. The liquidcrystal layer, acting as a $\lambda / 2$ retardation plate, changes the righthanded circular polarization into the left-handed one. Then, the converted left-handed circularly polarized light is selectively reflected by the second red cholesteric layer, $\lambda_{0} \approx 600 \mathrm{~nm}$, and on its way back crosses the anisotropic layer again. As a result, its left-handed circular polarization is reversed into the righthanded one, so that it penetrates the top layer without losses and comes out. Thus both left- and right-handed circular light is successfully reflected as it is observed for Plusiotis resplendens. ${ }^{[3,15]}$ The pitches of the cellulose cholesteric domains are chirped producing different reflected wavelengths on LCP and RCP light channels. Let us note that the exact thickness of the gap corresponding the $\lambda / 2$ retardation is not the sine quoi non condition for observing the reflection in the RCP channel. When the phase shift introduced by the nematic layer is different from $\lambda / 2$, the RCP reflection will not vanish but its amplitude will be smaller (see Figure S4 and the accompanying calculation in the Supporting Information). Taking into account the proposed model, the reflection spectra were numerically simulated by means of a $4 \times 4$ Berreman matrix. ${ }^{[24]}$ In order to perform the simulation the values of the nematic 5CB birefringence, ${ }^{[25]}$ the thickness and the pitches of the cholesteric domains as well as the gap height values (measured from the SEM images) were used. As shown in Figure 2I, the maximum of the reflected RCP and LCP channels' wavelengths are centered at the same values obtained experimentally, while the widths at half-height of the peaks are much thinner when compared with the experimental spectra. This can be attributed to the films' heterogeneities associated with the variation of the pitches of the two top layers of the CNC film, as well as the gap thickness' variation. The latter is not tuned thoroughly with the cholesteric domains' optical characteristics and does not act flawlessly as a half-wave retardation plate.

The use of a liquid crystal as an anisotropic $\lambda / 2$ retardation plate permits tuning of the reflected RCP and LCP light channels by changing the birefringence $\Delta n$, which can be achieved for instance by varying the temperature. In Figure 3 , the effect of the nematic to isotropic transition $\left(\mathrm{T}_{\mathrm{NI}}\right)$ is shown for reflected LCP light (Figure 3a,c,d) as well as for reflected RCP light (Figure $3 \mathrm{~b}, \mathrm{e}, \mathrm{f}$ ). While above $\mathrm{T}_{\mathrm{NI}}$ the LCP light reflection persists with an intense reddish color (Figure 3d) the RCP light reflection image becomes colorless (Figure 3f) (see Movie S2 and S3, Supporting Information). This behavior is an indication that the cholesteric CNC left-handed structure was permanently imprinted when the film was fabricated and remains indelible by the use of the LC in this temperature range. The schemes (Figure 3g,h) denote the liquid-crystal-layer transition below and above $\mathrm{T}_{\mathrm{NI}}$, this process being reversible. It is important to note that above $\mathrm{T}_{\mathrm{NI}}\left(T=34.5^{\circ} \mathrm{C}\right)$ the reflected LCP light wavelength shifts to higher values $\left(\lambda_{030.0{ }^{\circ} \mathrm{C}}=531 \mathrm{~nm}\right.$; $\left.\lambda_{034.5{ }^{\circ} \mathrm{C}}=562 \mathrm{~nm}\right)$. The wavelength reflection of the incident light on the cholesteric domains, along the cholesteric axis, can vary due to two parameters according to Equation (1): the average index refraction of the phase and the pitch. When a decrease of the index of refraction of the material occurs, the pitch of the nematic chiral phases increases due to the presence of an isotropic liquid between the layers. The effect of temperature was also observed in chiral nematic glasses impregnated with $8 \mathrm{CB}^{[26]}$ and the initial iridescence of the films was lost when the liquid crystal attains the isotropic phase. The different behavior observed for $\mathrm{CNC} / 5 \mathrm{CB}$ composites is mainly ruled by the anchoring of the liquid-crystal/cellulose system. ${ }^{[16]}$

Taking advantage of the external field stimuli-responsive LC layer, the system's birefringence can also be controlled by the application of an electric field. As can be seen in Figure 3i,j for the reflected LCP light channel, and in Figure $3 k, 1$, for the reflected RCP light channel, the electric field and thermal effects are similar: while the RCP light vanishes the LCP light channel reflection shifts to the red wavelength. When the electric field is applied, due to the positive dielectric anisotropy of $5 \mathrm{CB}$, the molecules of the nematic align along the electricfield direction inside the gap, schematically represented in Figure $3 m, n$.

The results simulated numerically are presented in Figure 3o. Predictably as the value of the birefringence vanishes the reflected RCP light channel is extinguished, while the reflected LCP light channel persists. However the experimental results (Figure 3p; see Figure S5a,b in the Supporting Information for spectra) only match these data trends for fields above a

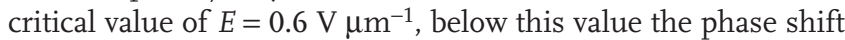
$\lambda / 2$ retardation plate is tuned by the electric field by adjusting the value of $\Delta n$ according to Equation (2). Movie S4 and S5 in the Supporting Information show the reversibility of the electric field in a film's region, which covers responsive and nonresponsive areas in order to enhance LCP and RCP channels' behavior. The nonresponsive area corresponds to heterogeneities of the film where the LC domain's thickness does not meet requirements of Equation (2). The POM reflection images shown in Figure $3 i-1$ are representative of the electrical-optical response of the film. The electro-optical cell cross-section showing the 

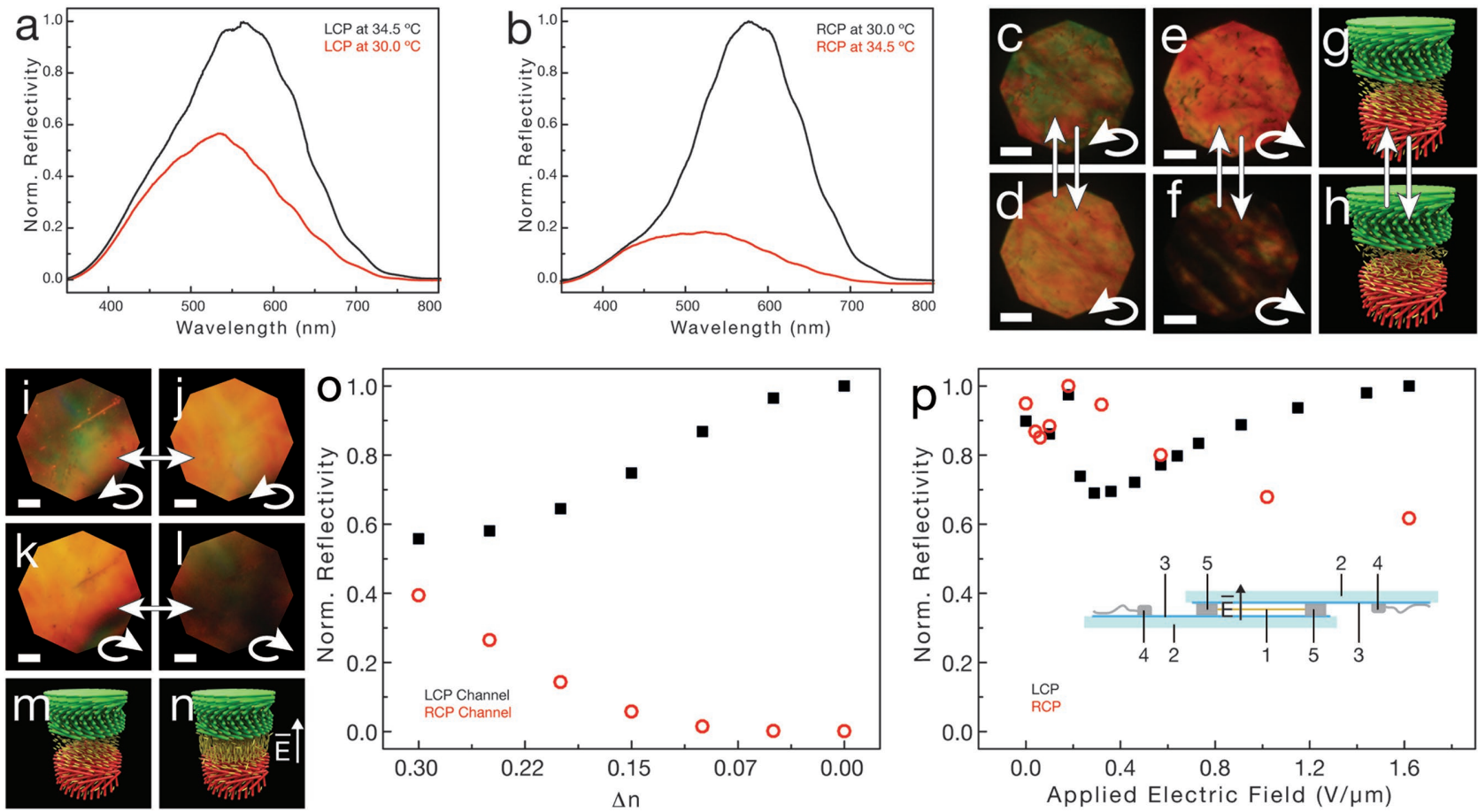

Figure 3. a-h) Thermal switching of reflected LCP and RCP channels in chiral CNC/liquid-crystal films. a,b) Plots representing the LCP and RCP channel responses, respectively, below $\left(30.0^{\circ} \mathrm{C}\right)$ and above $\left(34.5^{\circ} \mathrm{C}\right)$ the nematic-isotropic liquid-crystal transition. $\left.\mathrm{C}-\mathrm{f}\right)$ The reflection images for LCP and RCP channels, respectively, and g,h) the corresponding schematic cross-section presenting the anisotropic $(\lambda / 2$ plate) $(g)$ and the isotropic layer ( $h$ ), which develops above the nematic-isotropic transition inducing the reset of the RCP channel. i-p) Electric-field switching of LCP and RCP channels in chiral CNC/liquid-crystal films. i-I) Reflection microphotographs LCP (i) and RCP (k) channels at $E=0 \mathrm{~V} \mu \mathrm{m}^{-1}$ and at $E=1.6 \mathrm{~V} \mu \mathrm{m}^{-1}$ for both channels (j,I), showing the reflection decrease of RCP channel. $m, n)$ Schematic representation of the sample cross-section: $(n)$ shows the effect of the electric field $(\bar{E})$ applied perpendicular to the 5 CB positive dielectric nematic layer. o,p) Plot presenting the simulated curves using Berreman's method for different anisotropic layer birefringence (o), and the experimental values of the sample's reflectivity, for LCP and RCP channels, with the applied voltage (p). The inset in panel (p) shows a scheme of the electro-optical cell (1 - CNC/LC film; 2 - glass slide substrates; 3 - ITO and alignment layer; 4 - electric wires; 5 - spacers). The scale bars represent $100 \mu \mathrm{m}(\mathrm{c}-\mathrm{f})$ and $20 \mu \mathrm{m}(\mathrm{i}-\mathrm{I})$.

different components of the device is depicted in Figure $3 p$ (inset) highlighting the electric-field direction $(\overline{\mathrm{E}})$.

In summary, the macroscopic observation of the CNC films impregnated with the nematic liquid crystal under white light shows the reflection of reddish-greenish mixed tiny dots, which become blended in the overall hue depending on the view angle. When the pixelated sample is observed with circularly polarized light, islands with mainly segregated red and green colors appeared with higher vibrance, the colored dots being grouped in different regions of the sample. Our SEM results indicate that these regions correspond to cholesterics, which are at the top surface and directly below the gap regions, with different pitches. The presence of a liquid-crystal layer enhances the optical characteristics of the two first top cholesteric layers of the film, hindering the contribution from the bulk remaining $\mathrm{CNC}$ cholesteric domains. The self-assembly of the CNCs, which depends upon the conditions of the films' preparation, are crucial for the design of the layered structure that brings together the precise pitches for the exact band microgap heights. Moreover these gaps can be adjusted by the application of an external electric field.

We have shown, for the first time, that freestanding CNC solid iridescent films can reflect simultaneously right and left circularly polarized light, as it is observed for certain beetle cuticles, e.g., for Plusiotis resplendens, by simply inserting a nematic layer, acting as a $\lambda / 2$ retardation plate, between two left-handed cholesteric domains. Furthermore, we take advantage of the gaps existing along the film's cross-section to produce a new cellulose-based photonic structure consisting of an anisotropic layer sandwiched between two left-handed cellulose cholesteric domains with different pitches. The reflected RCP light channel response is highly dependent upon the birefringence of the liquid crystal, which can be reversibly tuned by the application of an electric field or by temperature variation. This method provides us with an unprecedented opportunity to take advantage of the "defects" observed in solid CNCs films' crosssections to produce new thermal and electro-photonic cellulosebased devices with response times in the order of milliseconds.

Finally, the wide availability and renewability of cellulose, combined with the simplicity of our approach, suggest a broad potential application of cellulose in advanced photonic devices.

\section{Experimental Section}

CNC Preparation: CNCs were obtained as described elsewhere ${ }^{[27]}$ from the acid hydrolysis of microcrystalline cellulose $(10 \mathrm{~g}$, Avicel $\mathrm{PH}-101$, derived from cotton as indicated by the supplier, Sigma-Aldrich, particle size $\approx 50 \mu \mathrm{m}$ ) with sulfuric acid (Sigma-Aldrich, 95-97\%) 
with an acid/solid ratio of $8.5: 1$, during $130 \mathrm{~min}$, under vigorous stirring, and quenched with ultrapure water (Millipore Elix Advantage 3 system). The resultant material was centrifuged with ultrapure water with consecutive cycles and the CNCs suspension collected for $\mathrm{pH}$ values between 1.9 and -3.9 . The product was dialyzed (Spectra/Por 4 membrane, molecular cut-off 12-14 kDa) against ultrapure water, for at least a month until a constant $\mathrm{pH}$ was reached. The suspension was treated with 3 consecutive cycles of $20 \mathrm{~min}$ of sonication over an ice bath using a UP400 S ultrasonic processor $(400 \mathrm{~W}, 24 \mathrm{kHz}$, Hielscher Ultrasonics $\mathrm{GmbH}$, with an energy input of $19 \mathrm{~kJ} \mathrm{~g}^{-1}$ ). The CNC suspension with a $1.94 \pm 0.05 \% \mathrm{w} / \mathrm{w}$ (gravimetrically determined) was used in its acid form $(\mathrm{pH}=3.5)$ and presents $0.85 \%$ (performed in triplicate) of total sulfur content determined by elemental analysis (with a Thermo Finnigan-CE Instruments Flash EA 1112 CHNS series, $\pm 0.3 \%$ ) equivalent to $4.39-\mathrm{OSO}_{3} \mathrm{H} / 100$ anhydroglucose units, according to the formula $\mathrm{C}_{6} \mathrm{H}_{10} \mathrm{O}_{5}-\left(\mathrm{SO}_{3}\right)$ and calculated from the equation $S(\%)=100 n \times S /[6 C+10 \mathrm{H}+(5+3 n) \mathrm{O}+n S] \cdot{ }^{[28]}$ The electrophoretic mobility was $-6.70 \times 10^{-8} \mathrm{~m}^{2} \mathrm{~V}^{-1} \mathrm{~s}^{-1}$ (zeta potential of $-129.4 \mathrm{mV}$, measure, at $25^{\circ} \mathrm{C}$, for $0.25 \% \mathrm{w} / \mathrm{w}, \mathrm{CNC}$ suspension, using a dynamic light scattering SZ-100 nanopartical series, Horiba, Ida, with a laser of $532 \mathrm{~nm}$ and Peltier system for temperature control). Nanoparticles with $152 \pm 65$ and $17 \pm 7 \mathrm{~nm}$ of length and width, respectively and aspect ratio $10 \pm 3$ were obtained (determined from 50 measures with Image), version $1.45 \mathrm{~s},{ }^{[29]}$ of the nanoparticles observed by SEM, see for instance Figure S2a,b, Supporting Information). These particle dimensions are in accordance to the ones described in the literature for similar hydrolysis time. ${ }^{[28]}$

CNC Films Preparation: Typically, the films were obtained by pretreating aqueous CNC $(1.9 \% \mathrm{w} / \mathrm{w})$ suspension with sonication ( $5 \mathrm{~min}$ ), in order to allow nanoparticles disaggregation. For polystyrene Petri dishes with $90 \mathrm{~mm}$ diameter, $100 \mathrm{~mL}$ of suspension were poured. Following slowly solvent evaporation at $20^{\circ} \mathrm{C}$ during 4 weeks, allowing the formation of a freestanding iridescent film with $52 \pm 1 \mu \mathrm{m}$ thickness (determined from 50 measures with a Mitutoyo digital micrometer). In all measurements error intervals are standard deviation.

Electro-Optical Cell Assembly: Cells were prepared by placing a section of CNC films $(\approx 12 \times 7.5 \mathrm{~mm}$, extracted from the central region of the films) between two $100 \Omega \square^{-1}$ indium tin oxide (ITO)-coated glasses $(15 \mathrm{~mm} \times 15 \mathrm{~mm} \times 0.7 \mathrm{~mm}$ ) and filled parallel to the films' surface, by capillarity, with 4-pentyl-4'-cyanobiphenyl (5CB - K15 Licristal, supplied by Merck). ITO-coated glasses were pretreated with poly(vinyl alcohol) aqueous solution (Sigma-Aldrich, $M_{\mathrm{w}}=80000 \mathrm{~g} \mathrm{~mol}^{-1} ; 5 \% \mathrm{w} / \mathrm{w}$ ) and rubbed in order to induce planar anchoring. The CNC samples, as showed in Figure 1, were placed apart from the glasses by using plastic spacers (101 $\pm 1 \mu \mathrm{m}$ thick, determined from 49 measures with a Mitutoyo digital micrometer) and the total cell thickness result in this case was $266 \pm 2 \mu \mathrm{m}$. Electrical contact copper wires were connected to the cell by using conductive silver cement (Leitsilber 200 by Ted Pella, Inc.).

CNC Film-LC Composite Preparation for SEM Observation: 4-Butyl-4'. cyanobiphenyl $(4 \mathrm{CB}$, supplied by Merck) was infiltrated within the CNC film mounted on a glass slide at $47.8{ }^{\circ} \mathrm{C}$ during $2 \mathrm{~h}$ using a hot stage Mettler FO82HT, coupled with a control processor Mettler F90. The glass supporting and the film composite were then fractured for crosssection observation.

SEM Observation: The average nanocrystal dimensions (length and width), film's pitch, gap, and height were determined from SEM. The images were obtained using a Carl Zeiss Auriga crossbeam (SEMfocus-ion-beam (FIB)) workstation instrument, equipped with an Oxford energy-dispersive X-ray spectrometer. Samples were mounted in aluminum stubs, coated with a thin carbon layer using a Q150T ES Quorum sputter coater. The acquisition was performed using an accelerating voltage of 2 or $5 \mathrm{kV}$ with $5.6-7.2 \mathrm{~mm}$ as working distance. Image) software, version $1.45 \mathrm{~s}$ was used to measure these entities and at least 30 measurements for each was done.

The SAXS data was collected on a laboratory-based SAXS instrument Xeuss from Xenocs. The X-rays are generated and focused on a microfocus source, Genix. The beam is collimated with two sets of scatterless slits. The films were measured on transmission mode and the scattering is collected on a Pilatus 300 detector. The intensity is expressed as a function of the momentum transfer modulus, $q$, defined as $q=4 \pi \sin \left(\theta^{\prime}\right) / \lambda$, where $2 \theta^{\prime}$ is the scattering angle and $\lambda$ is the radiation wavelength. The sample to detector distance is $0.9 \mathrm{~m}$ and the useful $q$ range is $0.01<q<0.45 \AA^{-1}$. The $2 \mathrm{D}$ scattering patterns were integrated using the program FOXTROT.

Spectroscopy, Photographs, Videos, and Optical Images: Photographs and videos of CNC film and CNC-5CB composite film were obtained with a Canon EOS 550D coupled with an EF-S $60 \mathrm{~mm}$ Canon macro-lens under visible light. Optical images/videos were obtained in reflection mode, by using an Olympus BX51 microscope, coupled with an Olympus DP73 CCD camera, and acquired with the Stream Basic v.1.9 Olympus software. A cold illumination source generated by a halogen lamp (KL 2500 LCD, Olympus) was used. The images were obtained with $\times 10$ or $\times 20$ objectives (Olympus, MPlanFL N) and automatically scaled by the software. To detect the reflection of right-handed and left-handed circularly polarized light, a quarter waveplate (U-TP137, Olympus) coupled with a polarizer was inserted in the optical path. The reflective wavelengths detected for LCP and RCP light channel were recordered with a Jobin Yvon monochromator $\mathrm{H} 10$ Vis, equipped with a photomultiplier mounted onto the microscope, and acquired with the Jobin Yvon Autoscan v 1.22 software. The reflective wavelengths of each sample, when submitted to temperature variations, were obtained by placing the samples in the hot stage Mettler FO82HT, coupled with a control processor Mettler F90, within the temperature range of 30.0-34.5, with an accuracy of $0.1{ }^{\circ} \mathrm{C}$, at $2{ }^{\circ} \mathrm{C} \mathrm{min}-1$, mounted on the microscope stage. POM images and reflective wavelength acquisitions during the application of an electric field to the samples were performed using an home-made system composed by a $20 \mathrm{MHz}$ function/arbitrary waveform generator (Agilent LXI 33220A) coupled with an amplifier Yamaha RX-V361. The values of the applied electric field were confirmed with a Protek 506 multimeter. All measurements were performed at room temperature using a $50 \mathrm{~Hz}$ frequency sinusoidal wave.

Reflectance Simulations: Theoretical simulations of the reflectance spectra were performed by means of a numerical simulation, based on the Berreman $4 \times 4$ method. This method uses a differential-matrix technique in order to solve the problem of reflection and transmission by cholesteric liquid crystals and other liquid crystals with continuously varying but planar ordering. ${ }^{[2]}$ The optical transmittance and reflectance spectra of media, such as nematic and cholesteric liquid crystals, can be computed using this method, by considering the medium as a stack of many thin parallel layers and by treating each one as if it is optically homogeneous. The spectra are determined considering the effects of refraction and multiple reflections of the electromagnetic waves, traveling through the stack of layers. ${ }^{[30]}$ The calculations were performed using an adaptation of a Python code written for the simple case of a single cholesteric-liquid-crystal layer. ${ }^{[31]}$ The reflectance spectra were obtained for a normal incidence of the incoming electromagnetic radiation waves, by imposing three variable parameters: the cholesteric pitch $P$ (193 and $170 \mathrm{~nm}$, for cholesteric domains 1 and 2, respectively), the thickness of each layer $h$ (by defining the number of half-turns: $2.89 \mathrm{~mm}$ and $2.55 \mathrm{~mm}$, for cholesteric domains 1 and 2, respectively, and $900 \mathrm{~nm}$ for the gap containing the nematic domain), and the liquid crystal's linear birefringence $\Delta n(0.3)$ by defining its ordinary - $n_{0}$ - and extraordinary - $n_{\mathrm{e}}$ - refractive indices (of 1.45 and 1.75, respectively). Average values of $P$ and $h$ were extracted from the obtained SEM images (Figure 2).

\section{Supporting Information}

Supporting Information is available from the Wiley Online Library or from the author.

\section{Acknowledgements}

This work was funded by FEDER funds through the COMPETE 2020 Program and National Funds through FCT - Portuguese Foundation 
for Science and Technology under the Projects Nos. POCI- 01-0145-FEDER007688 (Reference UID/CTM/50025), PTDC/FIS-NAN/0117/2014, and PTDC/CTM-BIO/6178/2014. S.N.F. acknowledges the Minister of Science, Technology and Higher Education for National Funds, European Social Funds and FCT for fellowship number SFRH/BDP/78430/2011. The authors also acknowledge FAPESP, CNPq, INCT-FCx from Brazil.

Received: July 6, 2016

Revised: September 20, 2016 Published online: November 9, 2016

[1] J. F. Revol, L. Godbout, D. G. Gray, J. Pulp Pap. Sci. 1998, 24, 146

[2] D. K. Gray, J-FOR 2013, 3, 6.

[3] S. Caveney, Proc. R. Soc. London Ser. B 1971, 178, 205.

[4] A. G. Dumanli, H. M. van der Kooij, G. Kamita, E. Reisner, J. J. Baumberg, U. Steiner, S. Vignolini, ACS Appl. Mater. Interfaces 2014, 6, 12302.

[5] H. de Vries, Acta Crystallogr. 1951, 4, 219.

[6] a) Q. Chen, P. Liu, F. Nan, L. Zhou, J. Zhang, Biomacromolecules 2014, 15, 4343; b) T. D. Nguyen, W. Y. Hamad, M. J. MacLachlan, Chem. Commun. 2013, 49, 11296; c) C. D. Edgar, D. G. Gray, Cellulose 2001, 8, 5.

[7] A. G. Dumanli, G. Kamita, J. Landman, H. van der Kooij, B. J. Glover, J. J. Baumberg, U. Steiner, S. Vignolini, Adv. Opt. Mater. 2014, 2, 646

[8] J. H. Park, J. Noh, C. Schütz, G. Salazar-Alvarez, G. Scalia, L. Bergström, J. P. F. Lagerwall, ChemPhysChem 2014, 15, 1477.

[9] D. Liu, S. Wang, Z. Ma, D. Tian, M. Gu, F. Lin, RSC Adv. 2014, 4, 39322.

[10] D. Gray, X. Mu, Materials 2015, 8, 7873.

[11] J. P. F. Lagerwall, C. Schütz, M. Salajkova, J. Noh, J. Hyun Park, G. Scalia, L. Bergström, NPG Asia Mater. 2014, 6, e80.

[12] X. Mu, D. G. Gray, Cellulose 2015, 22, 1103.

[13] J. Hwang, M. H. Song, B. Park, S. Nishimura, T. Toyooka, J. W. Wu, Y. Takanishi, K. Ishikawa, H. Takezoe, Nat. Mater. 2005, 4, 383.

[14] J. Majoinen, E. Kontturi, O. Ikkala, D. G. Gray, Cellulose 2012, 19, 1599.

[15] S. Kinoshita, S. Yoshioka, J. Miyazaki, Rep. Prog. Phys. 2008, 71, 076401.
[16] L. E. Aguirre, A. de Oliveira, D. Seč, S. Čopar, P. L. Almeida, M. Ravnik, M. H. Godinho, S. Žumer, Proc. Natl. Acad. Sci. USA 2016, 113, 1174 .

[17] P. G. de Gennes, Eur. Phys. J. E 2002, 7, 31.

[18] M. H. Song, B. Park, K. C. Shin, T. Ohta, Y. Tsunoda, H. Hoshi, Y. Takanishi, K. Ishikawa, J. Watanabe, S. Nishimura, T. Toyooka, Z. Zhu, T. M. Swager, H. Takezoe, Adv. Mater. 2004, 16, 779.

[19] H. Choi, J. Kim, S. Nishimura, T. Toyooka, F. Araoka, K. Ishikawa, J. W. Wu, H. Takezoe, Adv. Mater. 2010, 22, 2680.

[20] P. X. Wang, W. Y. Hamad, M. J. MacLachlan, Nat. Commun. 2016, 7, 11515

[21] X. Mu, D. G. Gray, Langmuir 2014, 30, 9256.

[22] S. Vignolini, P. J. Rudall, A. V. Rowland, A. Reed, E. Moyroud, R. B. Faden, J. J. Baumberg, B. J. Glover, U. Steiner, Proc. Natl. Acad. Sci. USA 2012, 109, 15712.

[23] a) M. Mitov, Adv. Mater. 2012, 24, 6260; b) M. Mitov, N. Dessaud, Nat. Mater. 2006, 5, 361.

[24] D. W. Berreman, J. Opt. Soc. Am. 1972, 62, 502.

[25] P. Oswald, P. Pieranski, in Nematic and Cholesteric Liquid Crystals, CRC Press, Taylor \& Francis Group, Boca Raton, FL, USA 2005, p. 65.

[26] a) A. P. Manning, M. Giese, A. S. Terpstra, M. J. MacLachlan, W. Y. Hamad, R. Y. Dong, C. A. Michal, Magn. Reson. Chem. 2014, 52, 532; b) M. Giese, J. C. de Witt, K. E. Shopsowitz, A. P. Manning, R. Y. Dong, C. A. Michal, W. Y. Hamad, M. J. MacLachlan, ACS Appl. Mater. Interfaces 2013, 5, 6854.

[27] a) D. Gaspar, S. N. Fernandes, A. G. de Oliveira, J. G. Fernandes, P. Grey, R. V. Pontes, L. Pereira, R. Martins, M. H. Godinho, E. Fortunato, Nanotechnology 2014, 25, 094008; b) J. F. Revol, H. Bradford, J. Giasson, R. H. Marchessault, D. G. Gray, Int. J. Biol. Macromol. 1992, 14, 170; c) E. D. Cranston, D. G. Gray, Biomacromolecules 2006, 7, 2522.

[28] a) X. M. Dong, J.-F. Revol, D. G. Gray, Cellulose 1998, 5, 19; b) W. Y. Hamad, T. Q. Hu, Can. J. Chem. Eng. 2010, 88, 392.

[29] Image/ website, http://imagej.nih.gov/ij/, accessed: October 2016.

[30] H. Wöhler, M. Fritsch, G. Haas, D. A. Mlynski, J. Opt. Soc. Am. A 1991, 8, 536.

[31] Olivier Castany, Source code python implementation of Berreman's 4x4 matrix method v3, Copyright 2012-2016, Olivier Castany, under GNU general public license; Copyright 2007, Free Software Foundation, Inc., http://fsf.org/, https://github.com/Berreman4x4/Berreman4x4; accessed: February 2016 